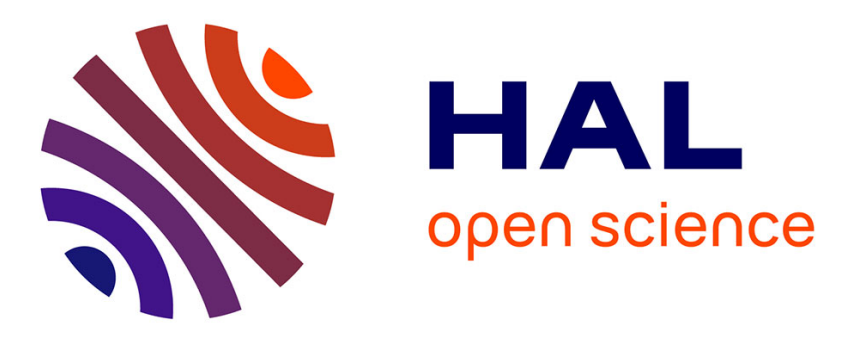

\title{
Anatomical subject validation of an instrumented hammer using machine learning for the classification of osteotomy fracture in rhinoplasty
}

Léo Lamassoure, Justine Giunta, Giuseppe Rosi, Anne-Sophie Poudrel, Jean-Paul Meningaud, Romain Bosc, Guillaume Haïat

\section{To cite this version:}

Léo Lamassoure, Justine Giunta, Giuseppe Rosi, Anne-Sophie Poudrel, Jean-Paul Meningaud, et al.. Anatomical subject validation of an instrumented hammer using machine learning for the classification of osteotomy fracture in rhinoplasty. Medical Engineering \& Physics, 2021, 95, pp.111-116. 10.1016/j.medengphy.2021.08.004 . hal-03430466

\author{
HAL Id: hal-03430466 \\ https://hal.science/hal-03430466
}

Submitted on 16 Nov 2021

HAL is a multi-disciplinary open access archive for the deposit and dissemination of scientific research documents, whether they are published or not. The documents may come from teaching and research institutions in France or abroad, or from public or private research centers.
L'archive ouverte pluridisciplinaire HAL, est destinée au dépôt et à la diffusion de documents scientifiques de niveau recherche, publiés ou non, émanant des établissements d'enseignement et de recherche français ou étrangers, des laboratoires publics ou privés. 


\section{Anatomical subject validation of an instrumented hammer using machine learning for the classification of osteotomy fracture in rhinoplasty}

Léo Lamassoure ${ }^{\mathrm{a}}$; Justine Giunta ${ }^{\mathrm{b}}, \mathrm{MD}$; Giuseppe Rosi ${ }^{\mathrm{a}}$; Anne-Sophie Poudrel $^{\mathrm{a}}$; JP.Meningaud, $\mathrm{MD}, \mathrm{PhD}^{\mathrm{b}}$; Romain Bosc, $\mathrm{MD}, \mathrm{PhD}^{\mathrm{b}, \mathrm{c}}$, Guillaume Haïat, $\mathrm{PhD}{ }^{\mathrm{a} *}$

${ }^{a}$ CNRS, Laboratoire de Modélisation et de Simulation Multi Échelle, UMR CRNS 8208, 61 avenue du Général de Gaulle, Créteil 94010, France

${ }^{b}$ Hôpital Henri Mondor, Plastic, Reconstructive, Aesthetic and Maxillofacial Surgery Department, 50 avenue du Maréchal de Lattre de Tassigny, 94000 Créteil, France

${ }^{c}$ INSERM U955 IMRB, Team 10, 94000 Creteil, France; UPEC Paris Est-Creteil University, Faculty of Medicine, F-94000 Creteil, France

* Corresponding Author:

Guillaume HAÏAT, $\mathrm{PhD}$

Laboratoire de Modélisation et de Simulation Multi Échelle, UMR CNRS 8208, 61 avenue du Général de Gaulle, 94010 Créteil, France

guillaume.haiat@cnrs.fr

Running Title: An instrumented hammer for osteotomies in rhinoplasty

Word Count: Abstract: 200 words (max 200); Manuscript 4163 words (max 5000)

Tables and Figures: 1 table, 7 figures

Keywords: Osteotomy, Rhinoplasty, Instrumented Hammer, Fracture

Journal: Medical Engineering and Physics 


\section{$1 \quad$ Abstract}

Osteotomies during rhinoplasty are usually based on the surgeon's proprioception to

3 determine the number and the strength of the impacts. The aim of this study is to determine

4 whether a hammer instrumented with a force sensor can be used to classify fractures and to

5 determine the location of the osteotome tip. Two lateral osteotomies were realized in nine

6 anatomical subjects using an instrumented hammer recording the evolution of the impact force.

$7 \quad$ Two indicators $\tau$ and $\lambda$ were derived from the signal, and video analysis was used to determine

8 whether the osteotome tip was located in nasal or frontal bone as well as the condition of the

9 bone tissue around the osteotome tip. A machine-learning algorithm was used to predict the

10 condition of bone tissue after each impact. The algorithm was able to predict the condition of

11 the bone after the impacts with an accuracy of $83 \%, 91 \%$, and $93 \%$ when considering a

12 tolerance of 0,1 , and 2 impacts respectively. Moreover, in nasal bone, the values of $\tau$ and $\lambda$

13 were significantly lower $\left(p<10^{-10}\right)$ and higher $\left(p<10^{-4}\right)$ than in frontal bone, respectively. This

14 study paves the way for the development of the instrumented hammer as a decision support 15 system. 


\section{Introduction}

Rhinoplasty is a common intervention in plastic surgery, representing around $9 \%$ of all

3 plastic surgery procedures according to the 2015 International Society of Aesthetic Plastic

4 Surgery (ISAPS) global survey [1]. It is usually realized to reshape the nose by modifying its

5 osseocartilaginous architecture and/or to correct breathing difficulties. Rhinoplasty is a complex intervention, even for an experienced surgeon [2] and it requires precise execution to obtain optimal results while respecting the aesthetic and functional aspects of the nose. Osteotomies are particularly crucial when taking care of deviated, hunchbacked noses, with a nasal bridge and / or a wide base [3], [4]. Osteotomies must be performed with caution to avoid any cosmetic or functional complications that could result in permanent deformities. minimal invasive approaches to limit visible scars. The surgeon positions and moves the osteotome to fracture bone tissue using his/her proprioception only (touch, hearing). The

14 development of a decision support system allowing to quantify bone properties around the osteotome tip would be of interest in order to help the surgeon adapt the impact strength throughout the surgical procedure. Such information could allow the surgeon to determine the progress of the osteotome through the different tissues, to anticipate an uncontrolled fracture and to obtain objective and quantified information on the osteotome pathway, which is often carried out blindly. Such device would be particularly interesting for lateral osteotomies aiming at separating the lateral slope of the bony nasal pyramid from its attachment to the maxilla, which are the most prone to variations [8].

Our group has been developing over the last few years a hammer instrumented with a

23 piezoelectric force sensor that allows measuring the variation of the force applied to the hammer during the several microseconds corresponding to each impact. This approach was originally 
1 designed to measure the stability of orthopedic implants such as acetabular cups or femoral

2 stems. Relevant indicators were derived from the measured signal [9]-[11] and tested in vitro

3 [12], ex vivo [13] and in anatomical subjects [14], [15].

More recently, the instrumented hammer was employed in the context of osteotomies,

5 first in vitro with composite materials and bone mimicking phantoms [16] and then ex vivo in

6 rabbit head samples [17]. The in vitro study [16] showed that the material of a sample could be

7 predicted with an estimated $89 \%$ prediction performance and that its thickness could be

8 estimated with an error lower than $10 \%$. The ex vivo study [17] then showed that a dedicated

9 indicator could be used to a) measure the displacement of the osteotome in the bone tissue

10 during a given impact and b) detect the arrival of the osteotome in frontal bone, which

11 corresponds to the end of the osteotomy.

The aim of the present study is to determine whether a hammer instrumented with a

13 piezoelectric force sensor can be used to retrieve information on the mechanical properties of

14 the tissue located around the tip of the osteotome during rhinoplasty. To do so, our approach

15 consisted in performing osteotomies in cadaveric subjects while using video analysis in order to validate the prediction of an algorithm based on the signal retrieved by the instrumented

17 hammer. More specifically, the objective is to determine whether a dedicated signal processing

18 technique can be used to follow the apparition of fractures during osteotomies performed with

19 the instrumented hammer. 


\section{Methods}

\section{1) Anatomical subjects:}

Our study was conducted in the anatomy laboratory of the Fer-à-Moulin surgical school (school of «Assistance Publique - Hôpitaux de Paris ») from December 2019 to March 2020 and was approved by its ethic committee (agreement \#9301/cnrs/05052021). The study involved nine anatomical subjects with an average age of $85 \pm 14$ years old. No chemicals were used for their preservation. They were stored in a cold room at $-18^{\circ} \mathrm{C}$ and then thawed at room temperature before the experiments..

\section{2) Surgical procedure:}

All experiments were carried out according to the same protocol by an experienced plastic surgeon. A subcutaneous infiltration of the entire nasal pyramid was performed with physiological serum, facilitating the subcutaneous detachment of the cartilages and nasal bones. The incisions were realized using an open rhinoplasty technique [18] in order to validate the displacement of the osteotome using video analysis (see paragraph 2.4 , video 1). The subcutaneous dissection began with a columellar incision, followed laterally by a bilateral marginal incision. The subcutaneous tissues were released from their cartilaginous attachments and raised, thus exposing the cartilaginous tip and the proximal part of the osseocartilaginous bump. A transverse incision from the glabella to the columella was made in order to allow soft tissue to be removed and the entire nasal pyramid to be exposed (Fig. 1). On each subject, two lateral ascending curved osteotomies of the "low to high" type [19] (video 1) were performed, leading to a total number of 18 osteotomies, each osteotomy having a total number of impact comprised between 21 and 49 . 


\section{3) Acquisition of the signal:}

The device used in this study was similar to the one used in $[16,17]$ and consisted in a 260g surgical mallet (32-6906-26, Zepf, Tuttlingen, Germany) equipped with a piezoelectric sensor having a measurement range up to $4.45 \mathrm{kN}$ (208C04, PCB Piezotronics, Depew, NY, USA) and calibrated by the manufacturer with an uncertainty of $\pm 1 \%$ (though the amplitude of the signal is not important in this study). The instrumented hammer was used to impact a 10 mm osteotome (reference \#32-6002-10, Zepf, Tuttlingen, Germany). Each signal corresponding to a given impact lasted several milliseconds. The force sensor was used to measure the time variation of the force during each impact. A data acquisition module (NI 9234, National Instruments, Austin, TX, USA) with a sampling frequency of $51.2 \mathrm{kHz}$ and a resolution of 24 bits was used to record, for each impact, the time variation of the force exerted on the osteotome over a duration of $2 \mathrm{~ms}$. The signals were transferred to a computer and recorded with a LabVIEW interface (National Instruments, Austin, TX, USA).

For each impact performed with the instrumented hammer, the recorded signal $s(t)$ corresponds to the evolution of the impact force as a function of time (video 1). The signal shape was qualitatively similar to the one obtained in the in vitro [16] and ex vivo [17] configurations. The same signal processing method as the one developed in vitro was applied to the signals corresponding to all impacts using Matlab (The MathWorks, Inc., Natick, Massachusetts, USA). Briefly, for the first and second peaks $(p=1,2)$ of each impact, the maximum peak amplitude $a_{p}$, the time of the center of the peak $t_{p}$, and the root mean square width $w_{p}$ were calculated using a Gaussian fit with a time window centered on the middle of the time window corresponding to $s(t)>a_{p} / 5$. Two indicators were calculated for each signal: the second peak time $\tau=t_{2}-t_{1}$ and the impulse ratio $\lambda=L_{2} / L_{1}$, where $L_{p}$ corresponds to the peak integrals approximated by: $L_{p}=(2 \pi)^{0.5} \cdot w_{p} \cdot a_{p}$. 


\section{4) Classification of the impacts:}

During all experiments, the position of the osteotome was recorded using a video camera (L-920M3, Spedal, Taiwan) in order to track fracture initiations and to classify each impact according to the bone status around the osteotome tip. After each impact, the bone-osteotome system (BOS) was considered to be in the "Fracture" (respectively "Bone") state when the video showed a fracture in front of the osteotome tip (respectively when the video showed the osteotome tip in direct contact with bone tissue); as illustrated in Fig 2. Moreover, for each osteotomy, the surgeon determined empirically (based on his proprioception) when the osteotome tip was assumed to be located in frontal bone, in which case the impacts were considered to be in the "Hard Bone" ("HB") state. Note that the surgeon did not have access to any information retrieved by the hammer during surgery in order to avoid any bias in the measurements.

Based on the classification of the BOS described above, the impacts were classified into four groups depending on the state of the BOS before and after the impact. When the BOS before and after the impact were in the "Fracture" (respectively "Bone") state, the impact was assumed to belong to the $F 2 F$ (respectively $B 2 B$ ) group. When the BOS before the impact was in the "Fracture" (respectively "Bone") state and the BOS after the impact was in the "Bone" (respectively "Fracture") state, the impact was assumed to belong to the $F 2 B$ (respectively $B 2 F$ ) group. Figure 3 illustrates schematically the different configurations.

A database was assembled, containing, for each of the 531 impacts, the corresponding state ("Bone", "Fracture", or "Hard Bone"), group (B2B, B2F, F2B, or $F 2 F)$, maximum peak force $\boldsymbol{F}$, and values of the indicators $\lambda$ and $\boldsymbol{\tau}$. Out of those 531 impacts, 6 were discarded because they corresponded to impacts where the osteotome had slipped out of the bone tissue (which would not happen during an actual surgery). 


\section{5) Machine-learning classifier:}

A dedicated iterative algorithm based on machine learning was developed in order to predict the state of the BOS for each impact in the database. The different osteotomy paths were analyzed separately, and for each of them, the first impact $I_{l}$ was not considered because it corresponds to the initiation of the osteotomy pathway. After $I_{1}$, the BOS was considered to be in the "Bone" state since we assumed that no fracture was initiated on the initial impact. For each impact $I_{n}$ ( $n>1$ corresponding to the impact number), four information were entered in the database:

- the state of the BOS for the impact $I_{n-1}$ ("Bone", "Fracture", "HB")

- the value of $\tau_{n}=\tau$ corresponding to the impact $I_{n}$

- the value of $\Delta \tau_{n}=\tau_{n}-\tau_{n-1}$, where $\tau_{n-1}$ corresponds to the value of $\tau$ for the impact $I_{n-1}$

- the value of $\lambda_{n}=\lambda$ corresponding to the impact $I_{n}$

The aim of the Support Vector Machine (SVM) classification algorithm [20] was to predict the state of the BOS after the impact $I_{n}$ (noted BOS $\left(I_{n}\right)$ in what follows). SVM models are machine-learning algorithms that can be used to separate the different groups of a dataset using hyperplanes, which are optimized to obtain prediction areas allowing to separate the points of the different classes. Here, a quadratic SVM model [20] was employed because it led to the best results compared to other types of SVM algorithms.

The algorithm is illustrated in Fig. 4 and is described in what follows. The first step was to decide whether the $\operatorname{BOS}\left(I_{n}\right)$ was in the " $H B$ " state or in other states. To do so, the parameters $(\tau, \Delta \tau)$ were considered and the output of the SVM algorithm was the equation $y=f_{H B}(x)$ in the $(\lambda, \tau)$ space separating impacts from the " $H B$ " state and from the other groups. When $\tau<f_{H B}(\lambda)$ (respectively $\tau>f_{H B}(\lambda)$ ), $\operatorname{BOS}\left(I_{n}\right)$ was classified as " $H B$ " (respectively in other groups). 
- If the $\operatorname{BOS}\left(I_{n}\right)$ was not considered to be in the " $H B$ " state, the algorithm was continued.

- If the $\operatorname{BOS}\left(I_{n-1}\right)$ was in the "Bone" state, the quadratic SVM algorithm was used in order to determine if the impact $I_{n}$ was in the $B 2 B$ or in the $B 2 F$ group. To do so, the parameters $(\tau, \Delta \tau)$ were considered and the output of the SVM algorithm was the equation $y=f_{B}(x)$ in the $(\tau, \Delta \tau)$ space separating impacts from the $B 2 B$ and from the $B 2 F$ groups. When $\Delta \tau>$ $f_{B}(\tau)$ (respectively $\Delta \tau<f_{B}(\tau)$ ), the impact was classified in the $B 2 F$ (respectively $B 2 B$ group). Such classification allows to determine the state of $\operatorname{BOS}\left(I_{n}\right)$ when the state of $\operatorname{BOS}\left(I_{n-1}\right)$ was considered to be in the "Bone" state.

- If $\operatorname{BOS}\left(I_{n-1}\right)$ was in the "Fracture" state, the same quadratic SVM algorithm was used in order to determine if the impact $I_{n}$ was in the $F 2 B$ or in the $F 2 F$ group. Again, only the parameters $(\tau, \Delta \tau)$ were considered. The $(\tau, \Delta \tau)$ referential was rotated by $45^{\circ}$ in order to be able to realize the interpolation, which led to a new set of coordinates $\left(\tau_{\text {rot }}, \Delta \tau_{\text {rot }}\right)$, given by:

$$
\begin{aligned}
& \tau_{\text {rot }}=\cos \left(45^{\circ}\right) \times \tau-\sin \left(45^{\circ}\right) \times \Delta \tau \\
& \tau_{\text {rot }}=\cos \left(45^{\circ}\right) \times \tau-\sin \left(45^{\circ}\right) \times \Delta \tau
\end{aligned}
$$

14 The output of the SVM algorithm was the equation $y_{r o t}=f_{F}\left(x_{\text {rot }}\right)$ in the $\left(\tau_{r o t}, \Delta \tau_{\text {rot }}\right)$ space 15 separating impacts from the $F 2 B$ and from the $F 2 F$ groups. When $\Delta \tau_{r o t}>f_{F}\left(\tau_{r o t}\right)$ (respectively $16 \Delta \tau_{\text {rot }}<f_{F}\left(\tau_{r o t}\right)$ ), the impact was classified in the $F 2 F$ (respectively $F 2 B$ ) group. Such 17 classification allows to determine the state of $\operatorname{BOS}\left(I_{n}\right)$ when the state of $\operatorname{BOS}\left(I_{n-1}\right)$ was 18 considered to be in the "Fracture" state. 
2 The algorithm described above was applied to all 531 impacts corresponding to 18 osteotomies

3 and the state of the BOS predicted by the algorithm was compared to the state of the BOS

4 observed with the video tracking analysis. For each impact, we determined whether and the

5 results obtained with the algorithm and with the video tracking analysis agreed and the error

6 was analyzed as a function of the predicted bone state. We also compared the two results when

7 considering a tolerance of one and two impacts for the prediction, which is justified by the fact

8 that several consecutive impacts were sometimes necessary to detect some fractures.

\section{Results}

\section{1) Signals measured}

Two typical signals corresponding to an impact realized in nasal bone (grey line) and in frontal bone (black line) are shown in Fig. 5, with the corresponding values of $\tau$. The value of $\tau$ obtained in frontal bone is significantly lower than the one obtained in nasal bone.

An ANOVA analysis carried out for all osteotomies showed that the values of $\tau$ (respectively of $\lambda$ ) were significantly lower (respectively higher) when the osteotome was in frontal bone compared to when it is in nasal bone $\left(p<10^{-10}\right.$ and $p<10^{-4}$, respectively).

\section{2) Classification study}

The results obtained for the three classification studies are represented in Fig. 6.

Figure 6A shows the values of the parameters $(\tau, \Delta \tau)$ obtained for all 531 impacts. The 
1 surgeon proprioception, while the crosses correspond to all other impacts. The result of the

2 SVM classifier is indicated by the black line, which has the following equation:

$$
\mathrm{y}=f_{H B}(\mathrm{x})=6.665 \cdot x^{4}-17.01 \cdot x^{3}+15.007 \cdot x^{2}-4,664 \cdot x+0.669
$$

The $(\tau, \Delta \tau)$ space in Fig. 6A is separated by the black line defined above into the dark and light grey areas. The dark area corresponds to impacts $I_{n}$ for which the $\operatorname{BOS}\left(I_{n}\right)$ is in the " $H B$ " state, while the light grey area corresponds to impacts $I_{n}$ for which the $\operatorname{BOS}\left(I_{n}\right)$ is in all other groups.

Figure $6 \mathrm{~B}$ shows the values of the parameters $(\tau, \Delta \tau)$ corresponding to impacts $I_{n}$ realized when the $\operatorname{BOS}\left(I_{n-1}\right)$ is in the "Bone" state. The crosses (respectively dots) correspond to impacts belonging to the $B 2 F$ (respectively $B 2 B$ ) group according to the video analysis. The result of the SVM classifier corresponds to the black line, which has the following equation:

$$
\mathrm{y}=f_{B}(\mathrm{x})=0,052 \cdot x^{4}-0,188 \cdot x^{3}+0,235 \cdot x^{2}-0,125 \cdot x+0,295
$$

The $(\tau, \Delta \tau)$ space in Fig. 6B is separated by the black line defined above into dark and light grey areas, which corresponds to coordinates of impacts belonging to the $B 2 B$ and $B 2 F$ groups, respectively.

Figure $6 \mathrm{C}$ shows the values of the parameters $(\tau, \Delta \tau)$ corresponding to impacts $I_{n}$ realized when the $\operatorname{BOS}\left(I_{n-1}\right)$ is in the "Fracture" state. The crosses (respectively dots) correspond to impacts belonging to the $F 2 F$ (respectively $F 2 B$ ) group according to the video analysis. The result of the SVM classifier corresponds to the black line, which has the following equation in the $\left(\tau_{r o t}, \Delta \tau_{r o t}\right)$ space (see subsection 2.5): 


$$
\mathrm{y}=\mathrm{f}_{F}(\mathrm{x})=0,415 \cdot x^{4}-1 \cdot 672 \cdot x^{3}+0,882 \cdot x^{2}+1,991 \cdot x-0,893
$$

The $(\tau, \Delta \tau)$ space in Fig. 6C is separated by the black line defined above into the dark and light grey areas which therefore corresponds to coordinates of impacts belonging to the $F 2 F$ and $F 2 B$ groups, respectively.

\section{3) Prediction Algorithm}

The results obtained in the last subsection and shown in Fig. 6 were used in the algorithm described in subsection 2.6 in order to predict the state of the BOS after each impact. For a given osteotomy, Fig. 7A shows the state of the BOS obtained after each impact using the video analysis, while Fig. 7B shows the corresponding results obtained by the aforementioned algorithm. The stars (respectively the white and black dots) correspond to a BOS in the "Fracture" (respectively "Bone" and " $H B$ ") state after the corresponding impact. The errors of the algorithm are indicated with a triangle in Fig. 7B. As shown in Fig. 7, a good agreement is obtained between the prediction of the algorithm and the results obtained using the video analysis since a false prediction was obtained for 2 out of 27 impacts.

As shown in Table 1, when considering all 18 osteotomy pathways, the results obtained with the algorithm and with the video analysis were in agreement in $83 \%$ of the cases (442 out of a total of 531 impacts). Moreover, video analysis found the presence of a fracture which was not predicted by the algorithm in $10 \%$ of the cases (55 out of 531 impacts), a situation corresponding to the $3^{\text {rd }}$ impact in Fig. 7. Conversely, video analysis found that the BOS corresponded to "Bone", while a fracture was predicted by the algorithm in $2 \%$ of the cases (10 out of 531 impacts), a situation corresponding to the $11^{\text {th }}$ impact in Fig. 7 . The algorithm failed 
1 to predict a BOS corresponding to " $H B$ " in $2 \%$ of the cases ( 9 out of 531 impacts), while a false

2 prediction of " $H B$ " was made in $3 \%$ of the cases ( 15 out of 531 impacts).

The third and fourth columns of Table 1 show the comparison between the results

4 obtained with video analysis and with the algorithm described in subsection 2.6 when allowing

5 an error of one and two impacts between the two modalities, respectively. As shown in Table

61 , the performances of the algorithm are significantly better when allowing an error of one and

7 two impacts.

8

\begin{tabular}{|c|c|c|c|c|}
\hline & Correct & Fracture & Bone & HB \\
& Predictions & False Positive & False Positive & False Positive \\
\hline Actual & $442 / 531$ & $10 / 531$ & $64 / 531$ & $15 / 531$ \\
Performance & $(83 \%)$ & $(2 \%)$ & $(12 \%)$ & $(3 \%)$ \\
\hline Perf. with $\pm \mathbf{1}$ & $480 / 531$ & $1 / 531$ & $39 / 531$ & $11 / 531$ \\
Tolerance & $(91 \%)$ & $(0 \%)$ & $(7 \%)$ & $(2 \%)$ \\
\hline Perf. with $\pm \mathbf{2}$ & $496 / 531$ & $0 / 531$ & $25 / 531$ & $10 / 531$ \\
Tolerance & $(93 \%)$ & $(0 \%)$ & $(5 \%)$ & $(2 \%)$ \\
\hline
\end{tabular}

9

10 Table 1. Performance of the algorithm for the prediction of the state of the bone-osteotome

11 system after each impact. The second column shows the raw performances of the algorithm,

12 while the third and fourth column show the performances when allowing a tolerance of 1 and

132 impacts, respectively. 


\section{Discussion}

The originality of the approach described herein is to help the surgeon collect objective

3 information on the nature of the tissues located around the osteotome tip throughout the

4 osteotomy procedure, including i) the presence of a fracture at the osteotome tip and ii) the

5 arrival of the osteotome in frontal bone. Moreover, the operating time is not modified since the information can be given in real time and the instrument is not invasive since the instrumented hammer does not touch the patient's tissues. Since osteotomies in rhinoplasty are often performed blindly, such device could provide objective information about the osteotomy site, regardless of the technique used by the surgeon.

Rhinoplasty is recognized as a difficult surgical procedure with a long learning process

11 [2] for three main reasons: the nasal anatomy is very variable, the procedure must correct shape and function, and the final result must meet the patient expectations. Our approach could 13 therefore allow young surgeons to achieve a faster learning curve for rhinoplasty, which is 14 particularly steep, since 70 to 100 trials are usually required to start mastering the gesture [21]. In addition, each rhinoplasty is different because the surgeon must adapt to the patient's request and its intrinsic characteristics [22]. All these advantages make it possible to consider a future routine clinical use of such instrument. Rhinoplasty surgeons involved in teaching residents must balance the practical experience of a trainee while ensuring that such difficult maneuvers are carried out properly. Today, educational tools for surgical simulation using 3D printed models allow young surgeons to acquire the necessary experience more quickly and safely [23], [24]. Such learning technique

22 is increasingly in demand but remains expensive and is never similar to the real anatomy of the 23 operating room. By giving real-time control to the experienced surgeon, accessibility to 
1 rhinoplasty could be made easier for young trainees. In addition, objective data will be useful

2 to osteotome manufacturers to control the quality and performance of the devices they design.

Coupling the data retrieved with the instrumented hammer to navigation systems such as CT-scan data could also increase the precision of the surgical procedure. By comparing the density of the maxilla, nasal, and frontal bones with the data acquired intra-operatively using the instrumented hammer, it could become possible to locate precisely the osteotome tip, somewhat reaching the neuronavigation principle used in otorhinolaryngological surgery for the sinus, ear, and cranial surgery [25].

Hubert et al. [16] developed an analytical model in order to understand the physical determinants of the signals shown in Fig. 5. The results found in [16] indicated that $\tau$ decreased as a function of the rigidity of the material located around the osteotome tip, which was given by the material and by the thickness of the plate. The results were confirmed experimentally by considering 6 types of materials and various plate thicknesses. Similar results were obtained more recently in an ex vivo animal study [17] that found that $\tau$ also decreased when the rigidity of the sample increased, which could be explained by the fact that a higher rigidity leads to a higher resonance frequency of the BOS and thus to a lower value of $\tau$. The results found in the present study are in agreement with previous experimentations [16] because of the following explanation.

First, Fig. 6B shows that fracture initiation corresponds to values of $\Delta \tau$ higher than 0.3 ms, which can be explained by the sudden decrease of rigidity due to the fracture initiation.

Second, Fig. 6C shows that fractures disappear when both values of $\tau$ and of $\Delta \tau$ are low, which corresponds to an increase of rigidity of the BOS due to the fracture disappearance. 
Third, Fig. 6A shows that the arrival of the osteotome in frontal bone corresponds to

2 low values of $\tau$, which is consistent with previous results obtained in a rabbit model and may

3 be explained by the higher thickness and rigidity of frontal bone compared to nasal bone [26],

4 [27]. Consequently, low values of $\tau$ could be used to detect the arrival of the osteotome in frontal

5 bone and the end of the osteotomy pathway.

6 However, this study has several limitations. First, we only considered one osteotome and the

7 results are likely to depend on its geometry, which is left to future studies. Second, the bone mechanical properties of anatomical subject may be different from those of patients. However,

9 the surgeon proprioception was similar to the one obtained in the operating room. Third, the

10 decision boundaries shown in Fig. 6 and obtained with the classification algorithms that were

11 optimized in order to maximize the accuracy of the trained model (i.e., to minimize the total number of errors), led to a total number 55 Fracture false negatives and 10 Fracture false positives. However, in a clinical context, it may be preferable to reduce the number of Fracture

14 false negatives at the cost of more Fracture false positives in order to maximize patient safety. Therefore, the algorithm's parameters could be adjusted to better fit the surgeon's needs.

16 Fourth, the remaining errors between video analysis and the algorithm prediction may be explained by i) sudden variation of bone thickness, leading to changes of values of $\tau$ [16], ii) issues associated with the video analysis system and/or with the 3-D fracture path which may lead to difficulties in detecting the presence of a fracture, and iii) errors made by surgeon when

20 determining whether the impact belongs to hard bone. Note that the errors are significantly 21 reduced when allowing a tolerance in terms of impact, which can be explained by the difficulty 22 of detecting the presence of a fracture or the location of the osteotome tip. Moreover, the 23 surgeon's proprioception and video analysis were used to classify the impacts and constitute 24 subjective criteria (similarly as what is done in the clinic). Thus, further studies should look 
1 into defining objective criteria that support the results described here. Fifth, the progress of the 2 osteotome due to a given impact was not measured herein as it was done in rabbit model because 3 of the difficulty of determining the location of the osteotome tip in this 3D configuration with 4 a sufficient precision. Future studies should focus on the possibility of determining such 5 modification of the osteotome location.

6 


\section{$1 \quad$ 5. Conclusion}

The results obtained with anatomical subjects indicate that employing an instrumented

3 hammer in combination with machine learning techniques may be an interesting approach to

4 provide a decision support system to the surgeon in order to retrieve information on bone status

5 around the osteotome tip during rhinoplasty.

The significant difference of biomechanical properties of the frontal and nasal bone

7 allows to determine when the osteotome reaches the end of the osteotomy pathway. Moreover,

8 the instrumented hammer allows the prediction of fracture apparition and disappearance in front

9 of the osteotome tip (video 1). In a clinical context, such information is critical to allow

10 adaptation of the following impacts' strength in order to avoid propagation of an existing

11 fracture more than desired. Therefore, the instrumented hammer is a non-invasive decision

12 support system that could provide clinicians with relevant and objective information in order to

13 assist optimized surgical procedures without modifying the surgical protocol. 


\section{Acknowledgements}

Competing interests: None declared

Funding: This project has received funding from the European Research Council (ERC) under the European Union's Horizon 2020 research and innovation program (grant agreement No 682001, project ERC Consolidator Grant 2015 BoneImplant).

The authors would like to acknowledge the support of the "Prématuration programme" of the CNRS through the Osteome project.

Ethical approval: Approval from the ethic committee of the Fer-à-Moulin surgical school (school of «Assistance Publique - Hôpitaux de Paris ») 


\section{References}

[1] P. I. Heidekrueger, S. Juran, D. Ehrl, T. Aung, N. Tanna, and P. N. Broer, "Global Aesthetic Surgery Statistics: a Closer Look," Plast Surg Hand Surg, vol. 51, no. 4, pp. 270-274, 2017.

[2] Y. Saban, "Rhinoplasty: lessons from 'errors," "HNO, vol. 66, no. 1, pp. 15-25, 2018.

[3] R. K. Daniel, "Mastering rhinoplasty: a comprehensive atlas of surgical techniques with integrated video clips," Springer Sci. Bus. Media, vol. 127, no. 5, pp. 2116-2117, 2010.

[4] M. Z. Siemionow and M. Eisenmann-Klein, "Nasal reconstruction and aesthetic rhinoplasty," in Plastic and Reconstructive Surgery, Springer, 2010.

[5] E. J. Dobratz and P. A. Hilger, “Osteotomies,” Clin. Plast. Surg., vol. 37, no. 2, pp. 301$311,2010$.

[6] J. Layliev et al., "Incidence and Preoperative Risk Factors for Major Complications in Aesthetic Rhinoplasty: Analysis of 4978 Patients.," Aesthetic Surg. J., vol. 37, no. 7, pp. 757-767, 2017.

[7] M. A. Shiffman and A. Di Giuseppe, "Advanced aesthetic rhinoplasty: art, science, and new clinical techniques," Springer Sci. Bus. Media, 2013.

[8] M. Uraloğlu, G. Efe, and R. Karacal, "Lateral Osteotomy Fixation Technique in Rhinoplasty," Craniofac Surg, vol. 30, no. 7, pp. e600-e603, 2019.

[9] V. Mathieu et al., "Variation of the impact duration during the in vitro insertion of acetabular cup implants," Med. Eng. Phys., vol. 35, no. 11, pp. 1558-1563, 2013.

[10] A. Michel, R. Bosc, V. Mathieu, P. Hernigou, and G. Haiat, "Monitoring the press-fit insertion of an acetabular cup by impact measurements: Influence of bone abrasion," Proc. Inst. Mech. Eng. Part H J. Eng. Med., vol. 228, no. 10, pp. 1027-1034, 2014. 
[11] A. Michel, R. Bosc, R. Vayron, and G. Haiat, "In vitro evaluation of the acetabular cup primary stability by impact analysis," J. Biomech. Eng., vol. 137, no. 3, 2015.

[12] H. Albini Lomami et al., "Ex vivo estimation of cementless femoral stem stability using an instrumented hammer," Clin. Biomech., vol. 76, 2020.

[13] R. Bosc et al., "Influence of soft tissue in the assessment of the primary stability of acetabular cup implants using impact analyses," Clin. Biomech., vol. 55, pp. 7-13, 2018.

[14] A. Dubory et al., "A cadaveric validation of a method based on impact analysis to monitor the femoral stem insertion," J Mech Behav Biomed Mater, vol. 103, 2020.

[15] A. Michel, R. Bosc, F. Sailhan, R. Vayron, and G. Haiat, "Ex vivo astimation of cementless acetabular cup stability using an impact hammer," Med. Eng. Phys., vol. 37, no. 2 , pp. 80-86, 2016.

[16] A. Hubert, G. Rosi, R. Bosc, and G. Haiat, "Using an impact hammer to estimate elastic modulus and thickness of a sample during an osteotomy," J. Biomech. Eng., 2020, doi: 10.1115/1.4046200.

[17] L. Lamassoure, J. Giunta, G. Rosi, A.-S. Poudrel, R. Bosc, and G. Haiat, "Using an Impact Hammer to Perform Biomechanical Measurements during Osteotomies: Study of an Animal Model," J. Eng. Med.

[18] R. J. Rohrich and P. N. Afrooz, "Primary open rhinoplasty," Plast. Reconstr. Surg., vol. 144, no. 1, pp. 102e-117e, 2019.

[19] R. Tahamiler and M. Yener, "Lateral Osteotomy in Rhinoplasty," in Advanced Aesthetic Rhinoplasty, Berlin: Springer, 2013, pp. 387-399.

[20] C. Cortes and V. Vapnik, "Support-vector networks," Mach. Learn., vol. 20, no. 3, pp. 
273-297, 1995.

[21] A. Yeolekar and H. Qadri, "The learning curve in surgical practice and its applicability to rhinoplasty," Indian J. Otolaryngol. Head Neck Surg., vol. 70, no. 1, pp. 38-42, 2017.

[22] P. J. F. M. Lohius, Advanced caucasian and mediterranean rhinoplasty. Amsterdam: Kugler Publications, 2014.

[23] M. A. AlReefi et al., "Development and validation of a septoplasty training model using 3-dimensional printing technology," Int. Forum Allergy Rhinol., pp. 1-6, 2016.

[24] D. Zammit et al., "Step-specific simulation: the utility of 3D printing for the fabrication of a low-cost, learning needs-based rhinoplasty simulator," Aesthetic Surg. J., 2020.

[25] V. Palazzolo et al., "Removal of a frontal sinus osteoma and reconstruction by a custommade implant with neuronavigation assistance," Craniomaxillofac. Trauma Reconstr., vol. 11, no. 4, pp. 305-313, 2018.

[26] K. Frank et al., "Age and gender differences of the frontal bone: a computed tomographic (CT)-based study,” Aesthetic Surg. J., vol. 39, no. 7, pp. 699-710, 2019.

[27] M. R. Norton and C. Gamble, "Bone classification: an objective scale of bone density using the computerized tomography scan," Clin Oral Impl Res, vol. 12, pp. 79-84, 2001. 


\section{Figure Legends}

Figure 1. Experimental configuration considered for the osteotomies.

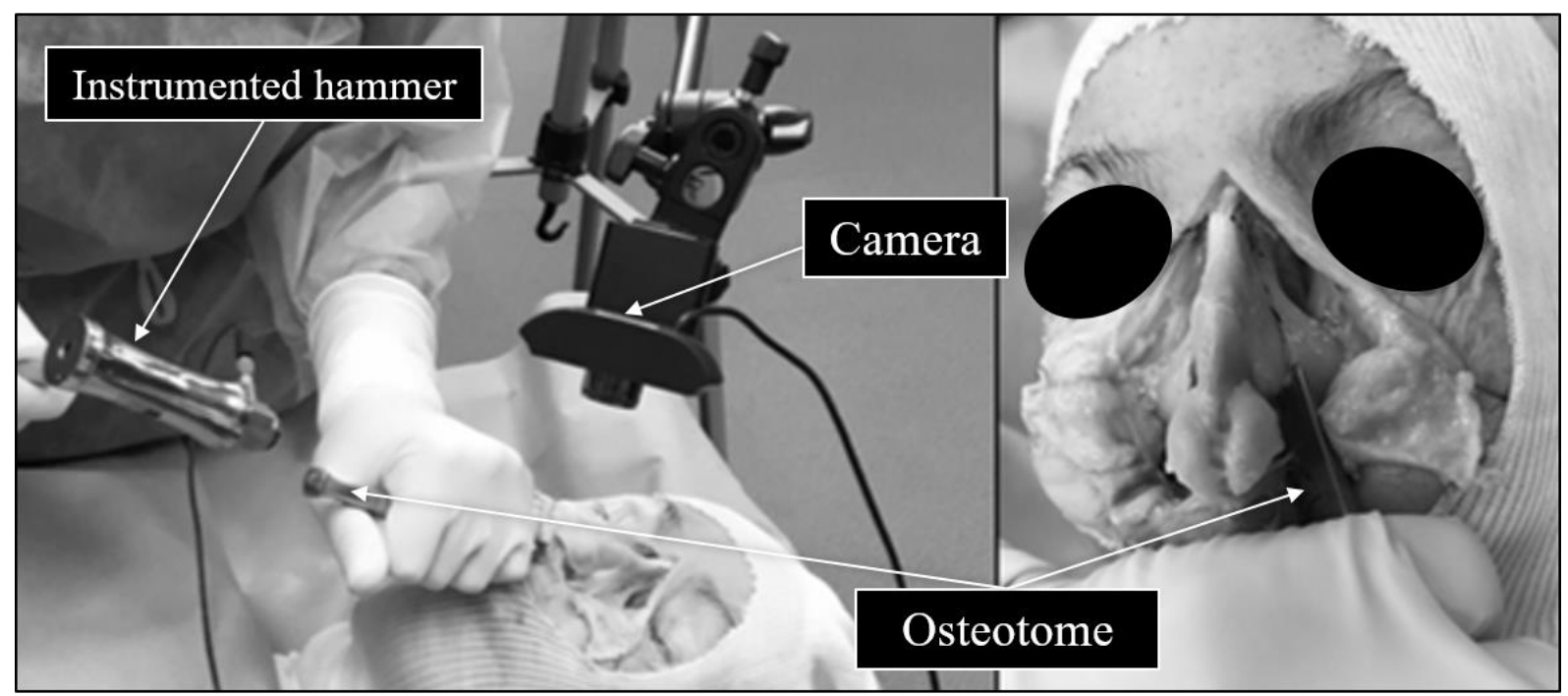

Figure 2. Image of the bone-osteotome system where (A) no fracture is present around the osteotome tip ("Bone" state) and (B) a fracture is located in front of the osteotome tip ("Fracture" state).
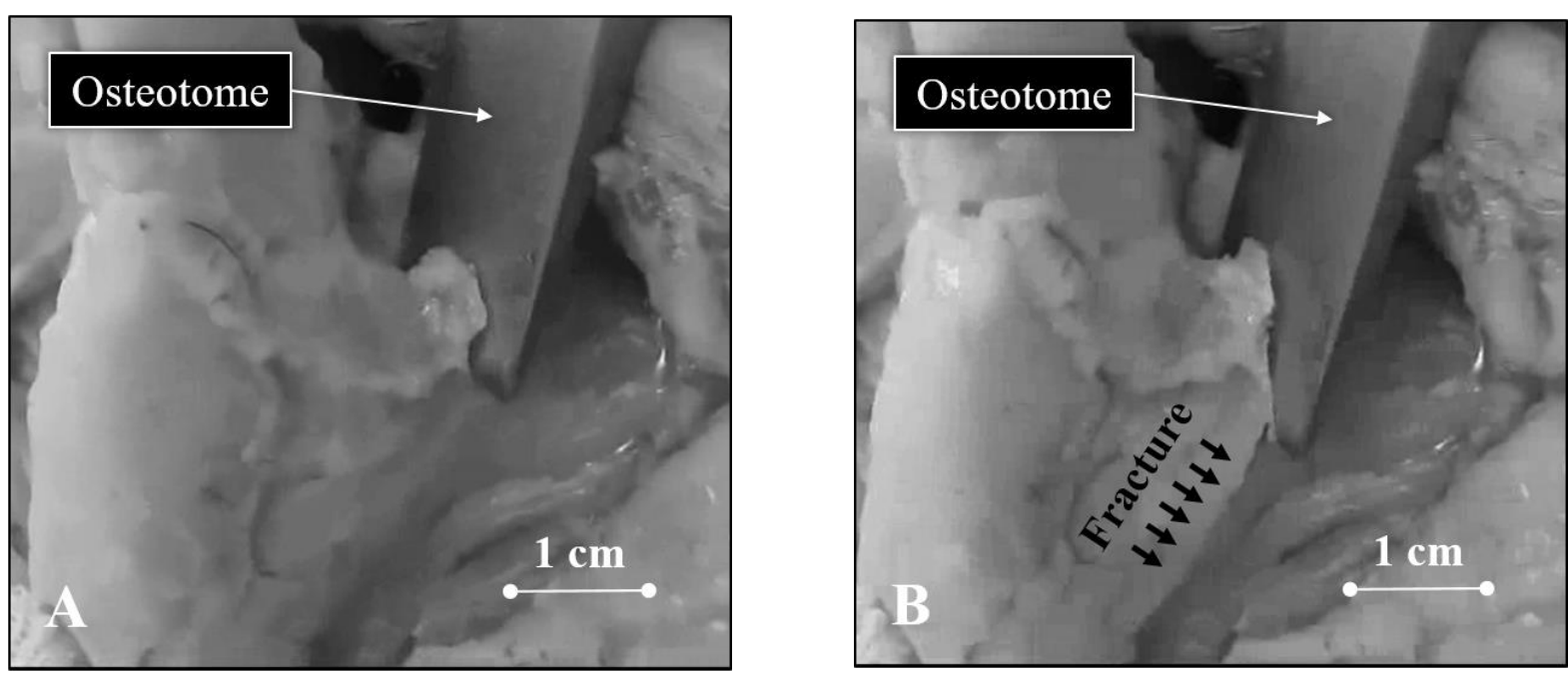
Figure 3. Schematic illustration of the different groups of impacts on the osteotome (light grey) in the bone tissue (dark grey) and fractures (white). A: Bone to Bone $(B 2 B), \mathrm{B}$ : Bone to Fracture $(B 2 F), \mathrm{C}$ : Fracture to Bone $(F 2 B)$, D: Fracture to Fracture $(F 2 B)$. The group of each boneosteotome system is indicated for each configuration.

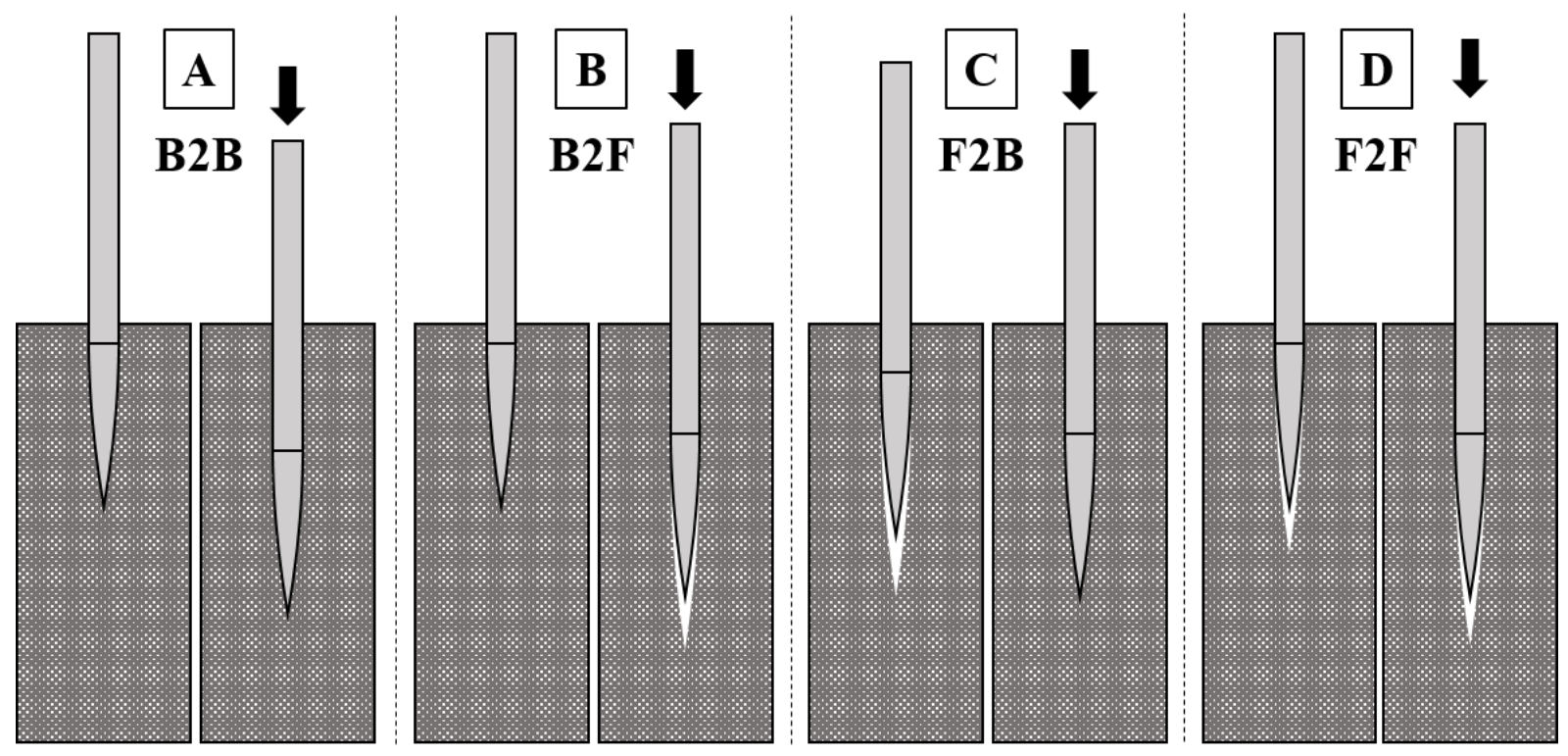


Figure 4. Schematic representation of the SVM based algorithm to predict the state of the boneosteotome system after each impact $I_{n}$. BOS $\left(I_{n}\right)$ indicates bone-osteotome system after the impact \#n. HB corresponds to Hard bone.

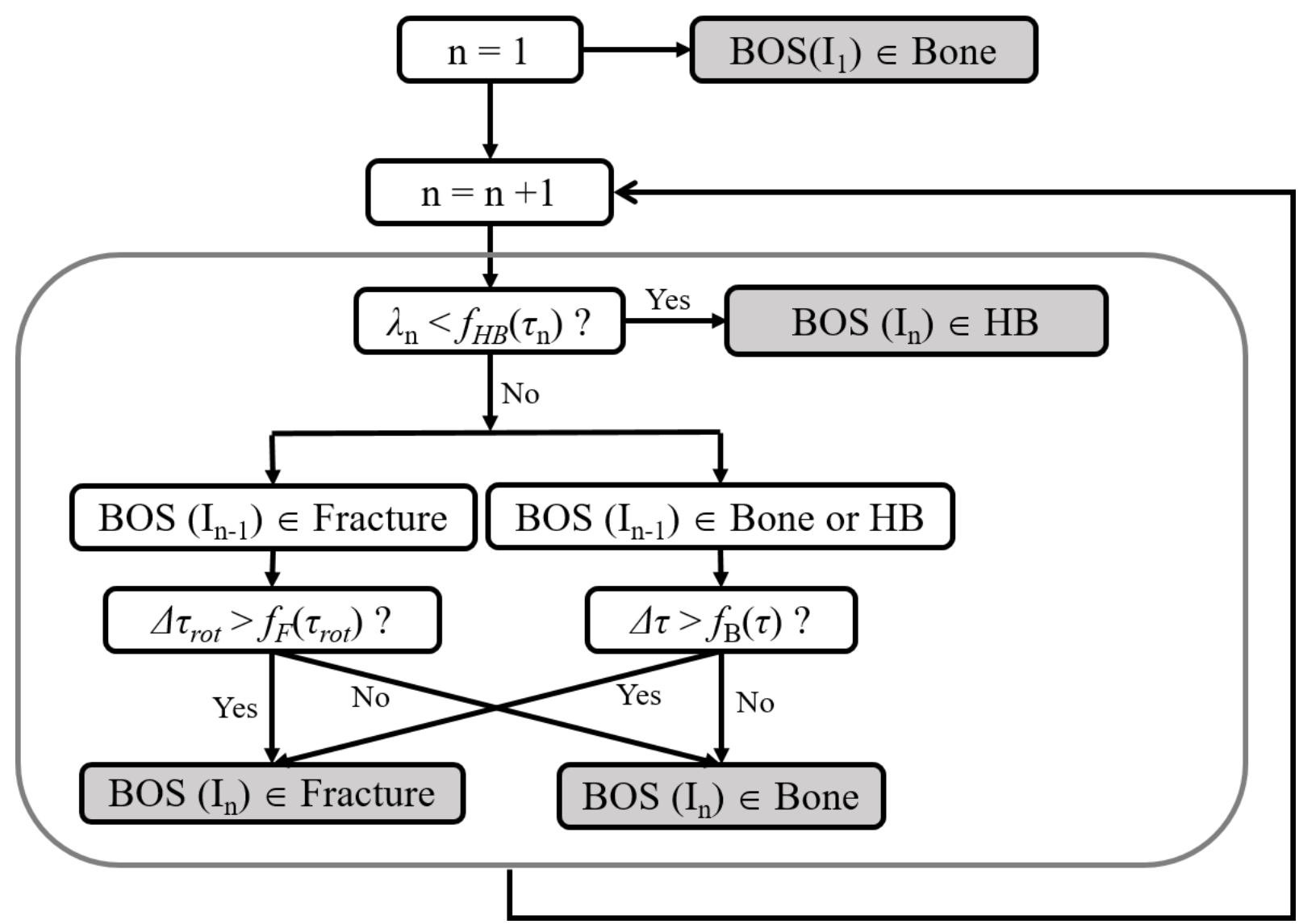


Figure 5. Example of the signals recorded for two different impacts of the instrumented hammer in the nasal bone and frontal bone, respectively. The values of the second peak time $\tau$ are indicated for each signal.

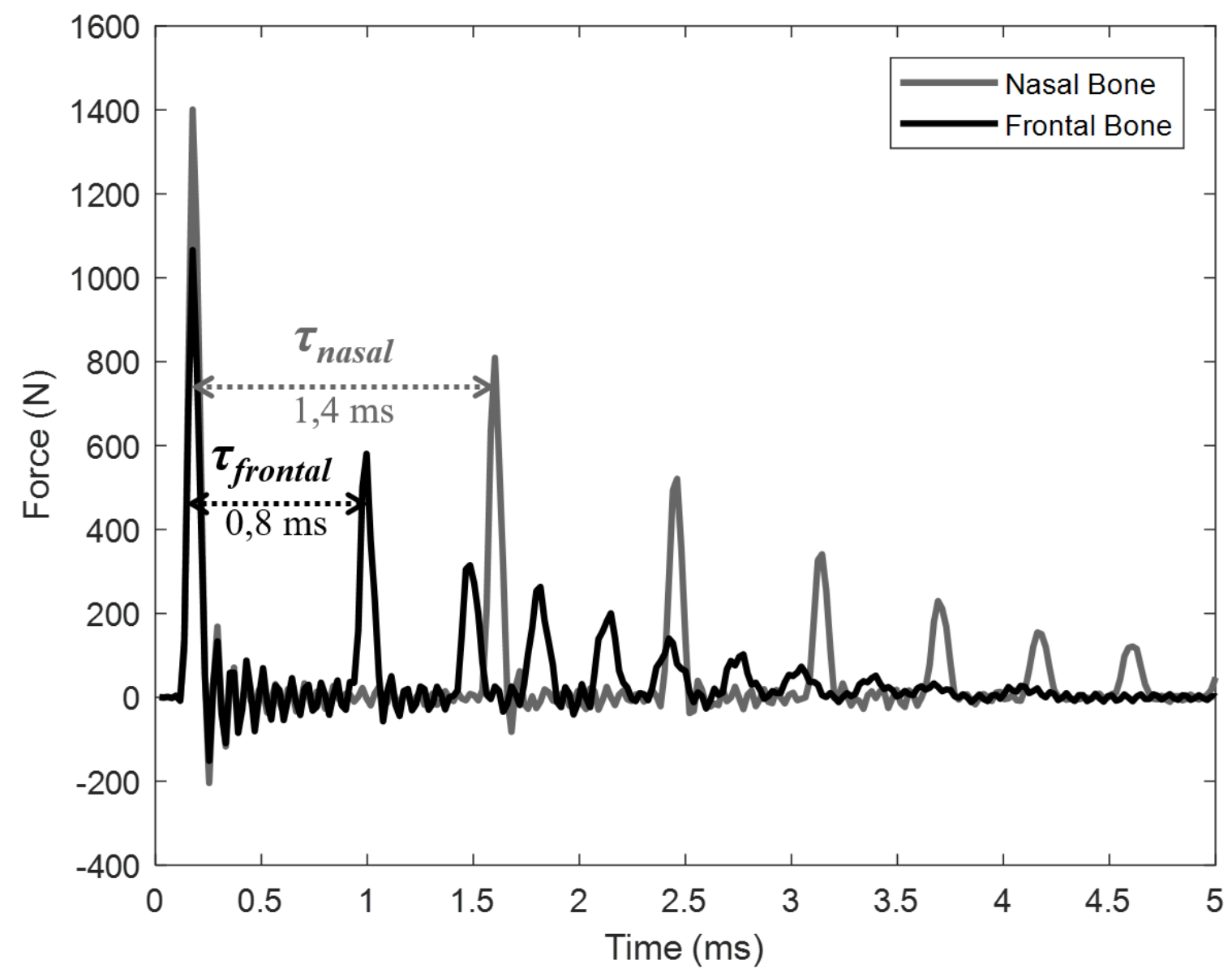


Figure 6. Results of the three classification studies. A: Classification of the BOS state corresponding to "Hard Bone" (dark grey) and to other states (light grey). B: Classification of the impact groups $B 2 F$ (dark grey) and $B 2 B$ (light grey). C: Classification of the impact groups $F 2 F$ (dark grey) and $F 2 B$ (light grey). For each classification, the decision boundary is plotted as a black line.
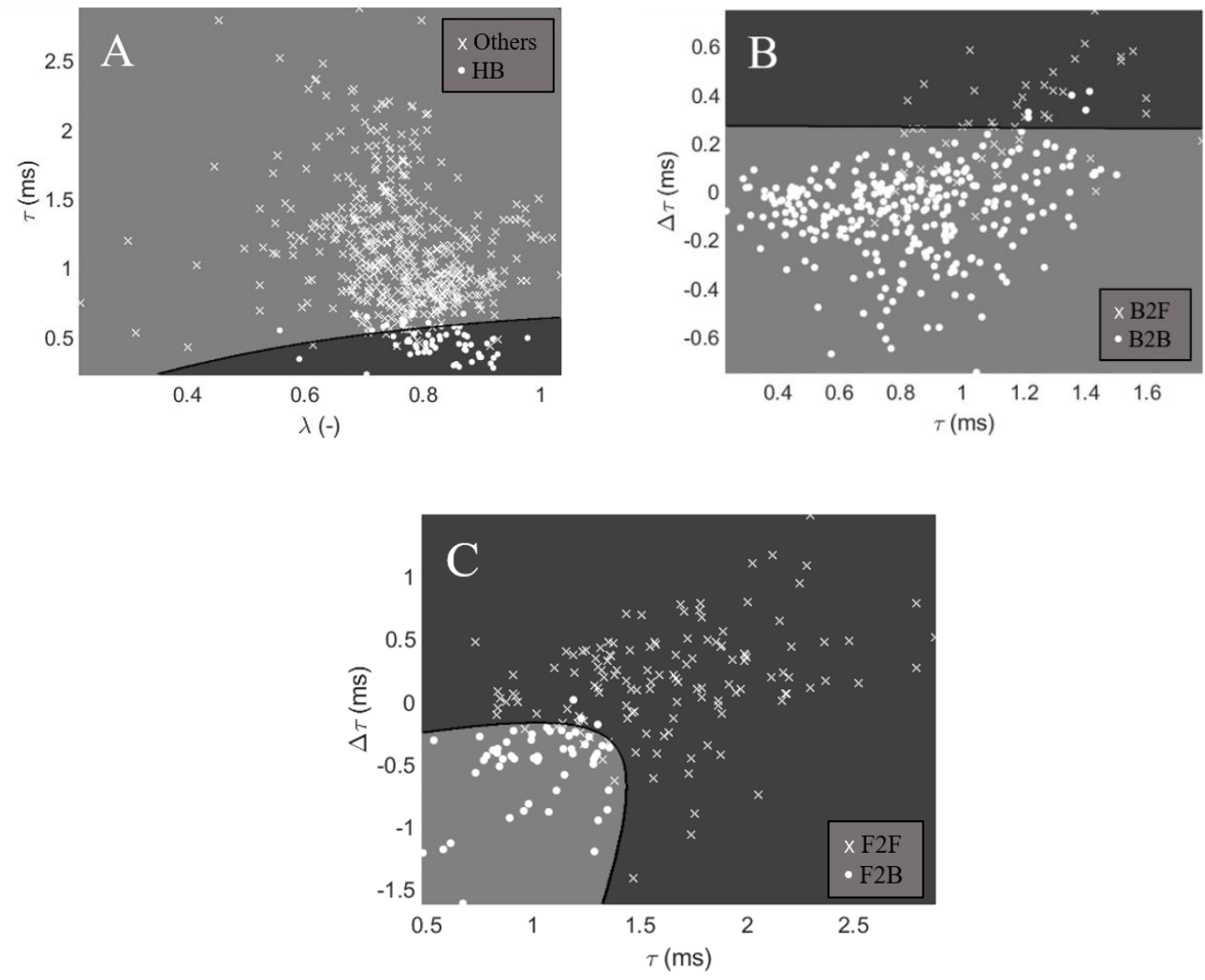
Figure 7. State of the bone-osteotome system obtained after each impact A: using the video analysis analysis and B: using the machine-learning algorithm. The errors are indicated with a triangle in $\mathrm{B}$.
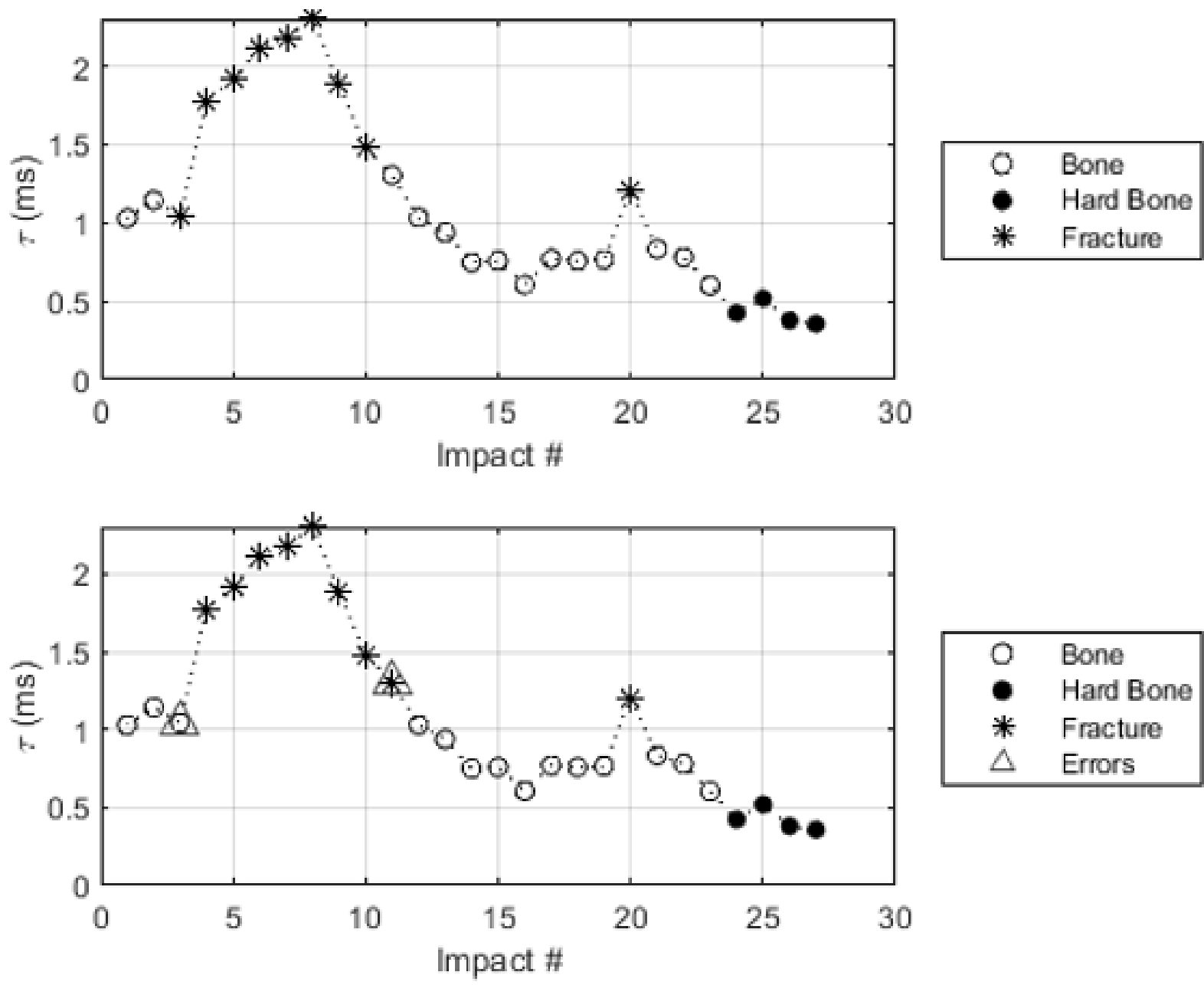

\begin{tabular}{|ll|}
\hline 0 & Bone \\
$\bullet$ & Hard Bone \\
$*$ & Fracture \\
$\triangle$ & Errors \\
\hline
\end{tabular}

